\title{
Conocimiento e importancia, que los profesionales de enfermería tienen sobre el consentimiento informado aplicado a los actos de cuidado de enfermería
}

\author{
Knowledge and importance nursing professionals have on informed consent applied to nursing care acts
}

\author{
Gloria Omaira Bautista-Espinel1* orcid.org/0000-0002-0551-4110 \\ Nelson Andrés Ardila-Rincón² orcid.org/0000-0002-2911-9502 \\ Juan Carlos Castellanos-Peñaloza ${ }^{3}$ orcid.org/0000-0001-7198-3102 \\ Yohana Gene-Parada 4 orcid.org/0000-0002-5412-0193
}

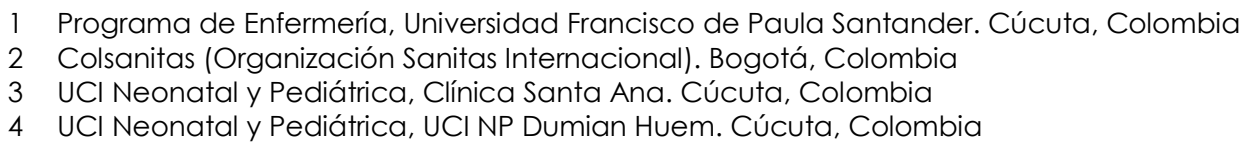

Fecha de recepción: Noviembre 05 - $2016 \quad$ Fecha de revisión: Mayo 26-2017 Fecha de aceptación: Agosto 11 - 2017

Bautista-Espinel GO, Ardila-Rincón NA, Castellanos-Peñaloza JC, Gene-Parada Y. Conocimiento e importancia, que los profesionales de enfermería tienen sobre el consentimiento informado aplicado a los actos de cuidado de enfermería. Univ. Salud. 2017;19(2):186-196. DOI: http://dx.doi.org/10.22267/rus.171902.81

\begin{abstract}
Resumen
Introducción: Los actos de cuidado que ofrece la Enfermería se fundamentan en la relación enfermero-paciente, el respeto por la dignidad y autonomía de las personas. Esto implica, comprender la importancia y el significado desde su esfera humana, aspecto que le otorga un sentido ético a las prácticas de cuidado. Este proceso reflexivo, amerita un adecuado conocimiento de las acciones que son necesarias para obtener el consentimiento informado. Objetivo: Identificar el conocimiento e importancia que los profesionales de enfermería tienen sobre el consentimiento informado, aplicado en los actos de cuidado, basado en la teoría de los patrones del conocimiento. Materiales y métodos: Estudio descriptivo, cuantitativo, transversal con una muestra de 221 profesionales de Enfermería, a quienes se les aplicó un cuestionario tipo escala de Likert; para el análisis se empleó estadística descriptiva, análisis factorial e inferencial. Resultados: Se evidenció poco conocimiento sobre el uso del consentimiento informado en los participantes. La variable importancia, se centra en el aspecto legal, administrativo e instrumental del consentimiento informado. Conclusión: Se debe fomentar el uso del consentimiento informado en actos de cuidado de la profesión. Así como los aspectos éticos, reforzar las formas de explicar los riesgos, beneficios y alternativas al paciente.
\end{abstract}

Palabras clave: Atención de enfermería; consentimiento informado; ética en enfermería; autonomía personal. (Fuente: DeCS, Bireme).

\begin{abstract}
Introduction: Nursing acts are based on the nurse-patient relationship, respect for the dignity and autonomy of people. This implies understanding the importance and meaning from the human sphere, which is an aspect that gives an ethical sense of care practices. This reflective process deserves adequate knowledge of the actions that are necessary to obtain the informed consent. Objective: Identify the knowledge and importance that nursing professionals have about the informed consent applied in the acts of care, based on the theory of knowledge patterns. Materials and methods: A descriptive, quantitative, cross-sectional study was conducted with a sample of 221 nursing professionals, who undertook a questionnaire type Likert scale. Descriptive statistics, factorial and inferential analysis were applied for the analysis. Results: It could be seen that there was little knowledge about the
\end{abstract}


use of informed consent in the participants. The variable called importance is focused on the legal, administrative and instrumental aspect of the informed consent. Conclusion: The use of informed consent should be encouraged in acts of care of the profession. The ways to explain the risks, benefits and alternatives to the patient must be reinforced as well as the ethical aspects.

Keywords: Nursing care; informed consent; ethics, nursing; personal autonomy. (Source: DeCS, Bireme).

\section{Introducción}

Es innegable la participación de enfermería en procedimientos y terapias invasivas, preventivas y curativas. Sus acciones forman parte de los planes de atención de salud que debe coordinar con el equipo interdisciplinario, en este proceso es reconocida su labor como el personal promotor del respeto por la autonomía del paciente. Acción, que va más allá de la verificación en el cumplimiento del proceso de obtención del consentimiento informado para los actos médicos, e incluso de otras profesiones. Para Moratilla et al., esta responsabilidad ética debe ser ejercida por cada grupo profesional, ya que el respeto por la autonomía del paciente es un compromiso que involucra a todos los miembros del equipo de salud, y no se debe seguir considerando como una tarea exclusiva de enfermería(1).

La incorporación del Consentimiento Informado (CI), por parte de enfermería a las actividades y procedimientos invasivos que realiza, le otorga sentido ético a las prácticas de cuidado, que va desde la colocación de catéteres, hasta la realización de procedimientos de cuidado directos, como curaciones, suministro de fármacos especiales $\mathrm{y}$ otras acciones que ameritan un adecuado conocimiento de los procesos graduales y permanentes, que son necesarios para su obtención. En este sentido la Asociación Colombiana de Facultades de Enfermería (ACOFAEN), en su Código de Ética plantea, que: "El reconocimiento de la autonomía del paciente se da ética y jurídicamente, con el respeto a la integridad de la persona, a los derechos del paciente"(2).

Entender la relevancia del CI, incluye comprender la importancia y el significado de la persona desde su esfera humana, aspecto que se ha transformado en una situación, que reclama la interacción dialógica, clara y respetuosa del enfermero con el sujeto de cuidado. No solo, como elemento de calidad, sino como un criterio ético que implica el respeto por la autonomía del paciente. A juicio de Alvarado(3) significa que algunos de los principios éticos y valores morales que predominan durante el proceso de atención de enfermería para cualquier paciente, en primera instancia, lidera la autonomía como derecho mismo del individuo de determinar sus propias acciones, de acuerdo con los planes que ha escogido, respetando sus convicciones $\mathrm{y}$, por ende, sus decisiones; todo lo anterior antes del consentimiento informado.

El Consejo Internacional de Enfermería (CIE), integra el consentimiento informado en los códigos deontológicos de la profesión a comienzos del presente siglo: años 2000 al 2005(4). En Colombia, el consentimiento informado en actos de cuidado de enfermería, se incorpora desde 2004; con la ley 911(5) y desde 1998 ACOFAEN elabora la "Guía para el desempeño del profesional de enfermería" y su código de ética, plantea las bases sobre los elementos del consentimiento informado, aplicables al ejercicio de la profesión(2).

A pesar de los actos normativos en torno al CI, en enfermería existen muchos interrogantes al respecto; como los expresados, por los profesionales de enfermería entrevistados por el Tribunal Nacional Ético de Enfermería de Colombia(6), algunos de estos son: ¿Qué planteamientos se han hecho acerca del consentimiento informado en enfermería?, ¿Hay varios tipos de consentimiento informado?, o ¿Cuál sería el más aplicable en enfermería?, ¿En cuáles intervenciones de cuidado se debe solicitar? y ¿Cómo hacerlo?, los cuales dan cuenta de los vacíos existentes en este campo.

En este sentido, Prieto de Romano destaca: "en la última década ha surgido un interés en la 
comunidad de enfermería por profundizar conceptualmente en CI y su aplicación en el cuidado". Además, existe un marco ético legal que fundamenta la exigencia de su aplicación en las diferentes áreas del ejercicio profesional; estos dos referentes son una guía para los enfermeros", pero al parecer no resuelven la totalidad de inquietudes sobre el tema ${ }^{(6)}$.

Rubio y Arias, al referirse sobre el planteamiento de Moreno y Durán, sostienen que "para conocer la disciplina de enfermería, es necesario comprender el concepto de "conocer" como un acto consciente, una forma particular e individual de visualizar las complejidades de una situación y de reunir recursos internos experimentados anteriormente para darle significado"(7).

Debido a la necesidad de adentrarse al significado del conocer en el personal de enfermería, sobre el consentimiento informado, se tomó como elemento orientador los patrones de conocimiento enfermero, que de acuerdo a lo expresado por Briñez ${ }^{(8)}$, sostiene que para Carper: "cada patrón da una orientación de los problemas y las preguntas de la disciplina, reconociendo que los cambios son inherentes al desarrollo del conocimiento en enfermería" aspecto, que permite adentrarse al objetivo del estudio, que busca: identificar el conocimiento e importancia que los profesionales de enfermería, tienen sobre el consentimiento informado aplicado en los actos propios del cuidado, basado en la teoría de los patrones del conocimiento.

Silva y Sorrell(10), manifiestan que Carper, describe y reconoce la existencia de cuatro patrones o formas de conocer en enfermería, las cuales se encuentran relacionados entre sí y que a la vez no son divisibles, estos son los patrones: empírico, ético, émico y estético. Según, Chinn citada por Landeros( ${ }^{(9)}$ : los patrones de conocimiento ético y empírico fluyen a través de la comunicación verbal y escrita, mientras los patrones de conocimiento personal y estético, fluyen a través de la conducta. Los cuatro patrones se revelan a través de la creación, de la expresión escrita y corporal de los procesos de cuidado enfermero.
Para Durán de Villalobos, “Carper definió que el cuerpo de conocimientos que da soporte a enfermería se manifiesta por patrones, o sea por medio de formas características de expresión externa e interna que dejan ver la manera como se piensa sobre un fenómeno. Los patrones no aumentan el conocimiento, más bien ponen su atención en el significado de conocer y sobre las clases de conocimiento que son de mayor ayuda para la disciplina de enfermería"(11).

En este contexto, los patrones se integran en las dimensiones de cuidado enfermero, desde la base del cocimiento y su expresión se refleja en aspectos como la abstracción mental y el conocimiento científico del patrón empírico, algo que Soto(12), citando a Chinn, describe como las formas de relacionarse con el mundo y la forma del conocimiento "más allá". El ser y el hacer que dan valor moral al patrón ético, en este patrón Soto(12) sostiene que "se refieren más bien al deber ser y al deber hacer. Va más allá de los juicios éticos, va hacia el hacer, de los motivos, intenciones y rasgos de carácter".

La identidad y personalidad que se relacionan con el fuero interno del enfermero dentro del patrón émico, es descrito por Soto, citando a Carper; como el más difícil de dominar ya que "implica un cocimiento ontológico del ser: ¿Quién soy? [...] enfermería usando la narrativa como formas de entender las formas de ser". Finalmente, el patrón que agrupa a los demás, que hace del cuidado, un acto artístico, que permite evidenciar la realidad, a través de representaciones creativas; dotadas de significado y sentido, están reflejados en el patrón estético, en palabras de Soto implica que: "la enfermera adquiere conocimiento estético a través de una experiencia sensible perceptualmente, singular y particular a través del reconocimiento empático con otros cuando entiende modos alternos de percibir la realidad. Los "entre" y "más allá" del conocimiento estético pueden ayudarnos a encontrar significados relevados en experiencias cotidianas"(12).

Lo anterior, permite dotar de contenido a la importancia que el personal de enfermería; puede dar al consentimiento informado. En este 
proceso es necesario, incorporar a la bioética y la ética, algo que Moratilla et al., sustentaron como "la necesidad que supone el "paso" desde el principio de beneficencia al principio de autonomía". Se hace necesario, integrar el concepto en el propio ser profesional en el que ningún miembro del equipo sanitario, se puede sentir exento. La enfermería debe sentir que por su carácter de cuidador, no puede ni debe mantenerse al margen de estos y otros principios de la bioética"(1).

\section{Materiales y métodos}

Este es un estudio cuantitativo de corte trasversal, recurre a métodos estadísticos que buscan establecer las relaciones entre las variables observadas (conocimiento e importancia) y los principales constructos a analizar, aplicando la teoría de los Patrones del Conocimiento de Enfermería (PCE), teniendo en cuenta los patrones: empírico, ético, émico y estético. Para el marco general del análisis estadístico, entre las distintas variables se realizó: análisis descriptivo, factorial e inferencial por conglomerados.

La población de estudio estuvo conformada por 221 profesionales de Enfermería, que se encontraban laborando en las instituciones de salud que tienen suscritos convenios de relación docencia servicio, con la Facultad Ciencias de la Salud de la Universidad Francisco de Paula Santander (UFPS), en el segundo semestre del 2015. Las entidades participantes del sector público fueron: dos empresas sociales del estado de baja complejidad y un hospital universitario de alta complejidad; del sector privado: tres clínicas de alta complejidad. Las instituciones están ubicadas en la Ciudad de Cúcuta y dos municipios del área metropolitana.

Para la selección de la muestra se realizó el cálculo sobre el número enfermeros que laboran en dichas IPS (Instituciones Prestadoras de Salud) públicas y privadas, que tienen convenio docencia servicios con la UFPS, para lo cual se realizó una selección no probabilística por conveniencia de todos los enfermeros que cumplieran con los criterios de selección y que accedieran a participar en el estudio.

El instrumento utilizado para la recolección de los datos corresponde a una encuesta sobre conocimiento e importancia del consentimiento informado en actos propios del cuidado de Enfermería elaborado por Bautista G,(13) en el año 2013. El cuestionario se subdivide en 40 ítems, con una escala para la variable importancia, cuatro sub-escalas para cada patrón del conocimiento que valora conocimiento. El instrumento fue validado en su contenido, mediante juicio de expertos en enfermería, bioética y pruebas paramétricas. La evaluación de propiedades métricas, se realizó mediante alfa de Cronbach, resultando un coeficiente de 0.838, en una prueba piloto con 21 sujetos. En el estudio de origen el instrumento se aplicó a 184 participantes. La tabulación y análisis de la información del presente estudio se realizó mediante el programa SPSS de IBM® versión19(14).

\section{Consideraciones éticas}

El estudio tuvo en cuenta los criterios éticos de investigación en humanos, contemplados en la Resolución 008430 de 1993(15). A los participantes se les solicitó autorización mediante documento de consentimiento informado, de otra parte, se garantizó la confidencialidad de la información, manteniendo las identidades de los participantes de forma anónima. Se aplicaron los criterios de protección de la información establecidos por el Comité de Ética Institucional.

Para el desarrollo de la investigación, se obtuvo el aval de cada uno de los comités de ética de las entidades participantes.

\section{Resultados}

La muestra estuvo conformada por 221 profesionales de Enfermería, la edad promedio osciló en el rango de 21 a 25 años. El género predominante fue el femenino con el $91,9 \%$. La distribución de la muestra por universidad y año de egreso, evidencia que el $78,8 \%$ de los profesionales participantes eran egresados de la 
Universidad Francisco de Paula Santander UFPS, en su mayoría del período comprendido entre 2011 y $2015(27,6 \%)$.

El nivel de estudio predominante fue el pregrado con un $62,9 \%$ y solo el $2,7 \%$ ha logrado culminar estudios de maestría. En las IPS públicas con un $38,2 \%$ se ubica el mayor número de profesionales de enfermería que han alcanzado un mayor nivel académico, en especialización y maestría. En las IPS privadas es mayor la concentración de profesionales con pregrado $(64,3 \%)$, seguido de especialización $(35,7 \%)$. En este sector no se encontró profesionales con nivel de maestría, aspecto que influye en la relevancia que se le puede dar al consentimiento informado, por la menor participación de estos profesionales en procesos de investigación (Tabla 1).

Tabla 1. Muestra analizada organizada por nivel de estudio / tipo de IPS

\begin{tabular}{|c|c|c|c|c|c|c|}
\hline \multirow[t]{3}{*}{ Nivel de estudio } & \multicolumn{4}{|c|}{ Tipo de IPS } & \multicolumn{2}{|c|}{ Total } \\
\hline & \multicolumn{2}{|c|}{ Pública } & \multicolumn{2}{|c|}{ Privada } & \multirow[b]{2}{*}{$\mathbf{F}$} & \multirow[b]{2}{*}{$\begin{array}{l}\% \text { Nivel } \\
\text { estudio }\end{array}$} \\
\hline & $\mathbf{F}$ & $\begin{array}{c}\% \\
\text { IPS }\end{array}$ & $\mathbf{F}$ & $\begin{array}{c}\% \\
\text { IPS }\end{array}$ & & \\
\hline Pregrado & 76 & 61,8 & 63 & 64,3 & $\begin{array}{r}13 \\
9\end{array}$ & 62,9 \\
\hline Especialización & 41 & 33,3 & 35 & 35,7 & 76 & 34,4 \\
\hline Maestría & 6 & 4,9 & 0 & 0 & 6 & 2,7 \\
\hline Total & 123 & 100 & 98 & 100 & $\begin{array}{r}22 \\
1\end{array}$ & 100 \\
\hline $\begin{array}{l}\text { \% participación } \\
\text { por sector }\end{array}$ & & 55,7 & & 44,3 & & 100 \\
\hline
\end{tabular}

Al clasificarse las instituciones por sector público y privado, no se encontraron diferencias significativas en cuanto la cantidad de participantes. El 55,7\% de los enfermeros están en instituciones del sector público y el $44,3 \%$ restante labora en el sector privado. La relación o diferencia entre sectores (público y privado) para el fomento del uso de $\mathrm{CI}$, no generó ningún tipo de condicionamiento y no hace la diferencia al momento de aplicarse en estas entidades participantes, aspecto que se evidencia en los contenidos de las guías y protocolos de enfermería. En ninguna de estas instituciones se incluye el uso del CI, dentro de los documentos de habilitación citados.

\section{Análisis factorial}

Se aplicó el Análisis de Varianza (ANOVA) de un factor en el que se tomó como variable dependiente las puntuaciones de cada patrón, y como factor o variable independiente los grupos etarios. Los resultados permiten afirmar que no existen diferencias significativas en los valores medios de los grupos de edad de las puntuaciones medias en todos los patrones: Empírico, Estético, Émico y Ético, así lo demuestra los valores de $p>0,05$ calculados $(\mathrm{p}=0,06 ; \mathrm{p}=0,617 ; \mathrm{p}=0,657$ y $\mathrm{p}=0,994)$.

Esto implica que la edad no es un determinante, que impida que el conocimiento sobre $\mathrm{CI}$, se pueda evidenciar desde los cuatro patrones. En este caso los profesionales, de todas las edades reconocen que el CI tiene una manifestación mediante una expresión escrita, que se puede incorporar a los procesos de cuidado enfermero y que para que el proceso se pueda implementar, se requiere de disposición que permita la apertura hacia el otro.

Al considerar el nivel educativo de los profesionales de enfermería encuestados, (pregrado, especialización y maestría), se encontró que las puntuaciones medias más elevadas corresponden al patrón de conocimiento émico y que las menos ponderadas corresponden al patrón ético. El patrón empírico y estético, entrecruzan sus valores medios aproximadamente en el punto 30 .

El nivel profesional se observa que el valor medio superior al punto 30, correspondió a los profesionales con formación en maestría, en tres de los cuatro patrones, exceptuando el ético. Se destaca la baja puntuación alcanzada por los tres niveles educativos para el patrón ético, versus la elevada ponderación del patrón empírico (Tabla 2).

Tabla 2. Puntuaciones medias en patrones de conocimiento por nivel de estudio

\begin{tabular}{lrrr}
\hline \multicolumn{1}{c}{$\begin{array}{c}\text { Patrón de } \\
\text { conocimiento }\end{array}$} & \multicolumn{3}{c}{ Nivel de estudio } \\
& Pregrado & Especialización & Maestría \\
\hline Patrón empírico & 30,1 & 30,4 & 30,7 \\
Patrón estético & 28,6 & 28,9 & 32,7 \\
Patrón émico & 30,4 & 29,2 & 33,3 \\
Patrón ético & 26,0 & 25,3 & 25,3 \\
\hline
\end{tabular}


Preocupa que el patrón ético relacionado con los conceptos de valor, el deber, las obligaciones éticas del ser y del hacer, esté en tan baja ponderación, ya que el discurso empleado para comunicar el CI es insuficiente y carece de los parámetros necesario para hacer posible la representación axiológica de $\mathrm{CI}$ en enfermería (al parecer valores como el respeto, la dignidad, tienden a confundirse con lo legal y normativo, mas no se identifican con el ser).

\section{Nivel de asociación de las puntuaciones de los patrones de conocimiento: empírico, estético, émico y ético}

El coeficiente de correlación de Pearson para los patrones de conocimiento se caracterizó por: la menor correlación que se presentó entre el patrón ético y el empírico, lo que implica que una variación en el patrón ético, no afecta al empírico y viceversa. Se observa que los demás patrones se encuentran relacionados entre sí en menor o mayor importancia, siendo el patrón émico, con el estético los de mayor correlación (Tabla 3).

Tabla 3. Coeficiente de Correlación $r$ de Pearson, para los patrones empírico, estético, émico y ético

\begin{tabular}{|c|c|c|c|c|c|}
\hline \multicolumn{2}{|c|}{ Correlaciones } & \multirow{3}{*}{$\begin{array}{r}\begin{array}{c}\text { Patrón } \\
\text { empírico }\end{array} \\
1\end{array}$} & \multirow{2}{*}{$\begin{array}{r}\begin{array}{r}\text { Patrón } \\
\text { estético }\end{array} \\
, 251^{* *}\end{array}$} & \multirow{2}{*}{$\begin{array}{r}\begin{array}{c}\text { Patrón } \\
\text { émico }\end{array} \\
, 208^{* *}\end{array}$} & \multirow{2}{*}{$\begin{array}{c}\begin{array}{c}\text { Patrón } \\
\text { ético }\end{array} \\
0,079\end{array}$} \\
\hline $\begin{array}{l}\text { Patrón } \\
\text { empírico }\end{array}$ & $\begin{array}{l}\text { Correlación } \\
\text { de Pearson }\end{array}$ & & & & \\
\hline & $\begin{array}{l}\text { Sig. } \\
\text { (bilateral) }\end{array}$ & & 0 & 0,002 & 0,24 \\
\hline & $\mathrm{N}$ & 221 & 221 & 221 & 221 \\
\hline \multirow[t]{3}{*}{$\begin{array}{l}\text { Patrón } \\
\text { estético }\end{array}$} & &, $251^{* *}$ & 1 &, $444^{* *}$ &, $187^{* *}$ \\
\hline & $\begin{array}{l}\text { Sig. } \\
\text { (bilateral) }\end{array}$ & 0 & & 0 & 0,005 \\
\hline & $\mathrm{N}$ & 221 & 221 & 221 & 221 \\
\hline \multirow[t]{3}{*}{$\begin{array}{l}\text { Patrón } \\
\text { émico }\end{array}$} & $\begin{array}{l}\text { Correlación } \\
\text { de Pearson }\end{array}$ & ,208 &, $444^{* *}$ & 1 & , $307^{* *}$ \\
\hline & $\begin{array}{l}\text { Sig. } \\
\text { (bilateral) }\end{array}$ & 0,002 & 0 & & 0 \\
\hline & $\mathrm{N}$ & 221 & 221 & 221 & 221 \\
\hline \multirow[t]{3}{*}{$\begin{array}{l}\text { Patrón } \\
\text { ético }\end{array}$} & $\begin{array}{l}\text { Correlación } \\
\text { de Pearson }\end{array}$ & 0,079 &, $187^{* *}$ & $307^{* *}$ & 1 \\
\hline & $\begin{array}{l}\text { Sig. } \\
\text { (bilateral) }\end{array}$ & 0,24 & 0,005 & 0 & \\
\hline & $\mathrm{N}$ & 221 & 221 & 221 & 221 \\
\hline
\end{tabular}

Esto permite inferir, que al momento de aplicar el $\mathrm{CI}$, el enfermero debe comprender las creencias y comportamientos que emanan desde su interior y también desde el punto de vista del sujeto de cuidado, es decir, mirar desde dentro, a quien da y tiene en cuenta a quien recibe, sin desatender que la forma particular como el enfermero realice el abordaje del CI, será desde lo estético, la forma como este exprese el arte del cuidado. Es necesaria una relación entre el contexto del acto de cuidado y el abordaje del problema que implica la aceptación o no, del acto por parte del paciente, que dependerá de la forma particular como el enfermero realice los procesos para la obtención del CI.

\section{Análisis inferencial por conglomerados}

Como procedimiento estadístico se partió del conjunto de datos (preguntas), que contenían información sobre los cuatro PCI, y se evaluó su relación con las variables, conocimiento e importancia dentro de la muestra de estudio. Se reorganizó la información en grupos relativamente más homogéneos, a los que se denominan conglomerados (clúster). Una vez se establecieron distancias o similitudes, existentes entre cada grupo por PCI se observó cuáles eran las preguntas más próximas en cuanto a esta distancia o similitud.

Estos formaron nuevos grupos durante el proceso, que con el apoyo del software se evaluó; la distancia entre el nuevo grupo formado, y las variables del estudio. Para esto se tomó la distancia máxima en cada grupo y la mínima distancia entre las preguntas. Seguidamente, surgió la representación gráfica de cada nueva clasificación, en un árbol de clasificación llamado dendograma, que resume el proceso de agrupación originado mediante análisis de clúster y que agrupó los ítems similares mediante enlaces, cuya posición en el diagrama está determinada por el nivel de similitud/diferencia entre las variables.

\section{Conglomerado del patrón empírico}

En el análisis por conglomerado, sobresale el enlace promedio entre grupos que integran la afirmación: "los protocolos en enfermería deben incluir las intervenciones y tipo de CI que requieren", unido a los reactivos que están relacionados con los acto, que son aceptados y 
permitidos por el paciente tales como: "el derecho a la libre escogencia dentro de varias opciones". En la esfera profesional el clúster se combinó con la afirmación: "el personal de enfermería responde a la necesidad de información del paciente $y$ no se limita únicamente a sus conocimientos"; a mayor distancia quedaron los reactivos que se relacionan con el cuidado y tratamiento. Estos aspectos se combinaron y permitieron la reclasificación nominal del clúster, bajo la denominación: Derecho y Fundamentos Procedimentales, que son necesarios conocer para aplicar el CI en el cuidado y tratamiento (Figura 1.)

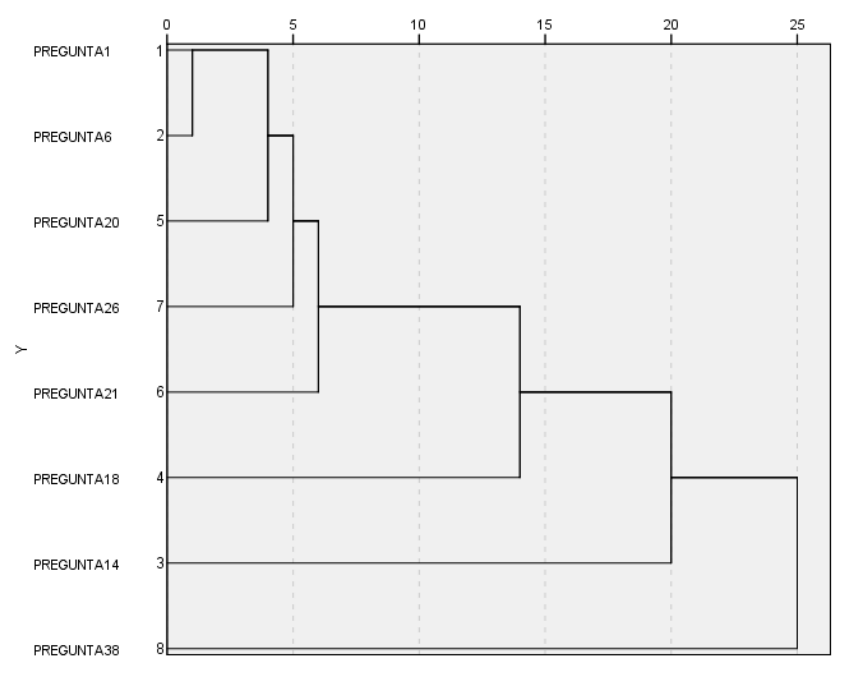

Figura 1. Dendrograma de enlace promedio, patrón empírico

Preguntas

1. El paciente tiene derecho a la libre escogencia sobre de los actos de cuidado que enfermería le pueda ofrecer. 6. El respeto por el principio de autonomía implica respetar la libre determinación del paciente. 20. Los protocolos de cuidado de enfermería, deben incluir: ¿en qué intervenciones se requiere el CI? y ¿qué clase de CI aplicar?. 26. Considero que algunos enfermeros (as) han interpretado que el CI, es un instrumento de protección del profesional ante posibles demandas por parte del paciente. 21. Tengo claridad conceptual y teórica sobre los actos del cuidado de enfermería que requieren solicitud $\mathrm{CI}$, ya sea verbal o escrito. 18. El CI, en enfermería se solicita en todas las acciones de cuidado tanto en el área clínica como en la comunitaria, sean invasivas o no. 14. El CI, escrito en enfermería solo se obtiene para procedimientos de tipo invasivo. 38. Considero que las enfermeras(os), deben responder a las necesidades de información, evaluar las necesidades del paciente, y limitarse únicamente a sus conocimientos profesionales.

\section{Conglomerado del patrón estético}

Este patrón adquiere significado en cada comportamiento individual del paciente, sobre el arte y representación que el acto de enfermería brinda a las acciones del cuidado. El análisis de elementos guarda relación con "la manera de informar y gestionar el cuidado, implica el adecuado fomento del CI escrito".

En un segundo momento encierra aspectos referentes a: "que el paciente firme el $\mathrm{CI}$, no implica la comprensión total de la información, si ésta no se explica claramente". Arrojando el análisis final de que los "Deberes de enfermería es informar el derecho a su sutonomía" al hablar del paciente. En este caso, se relacionan con los clúster "Operacionalización del uso del CI" y la "Gestión de la aplicación del CI", aspectos que fueron considerados como de tipo "Administrativo del CI" (Figura 2.)

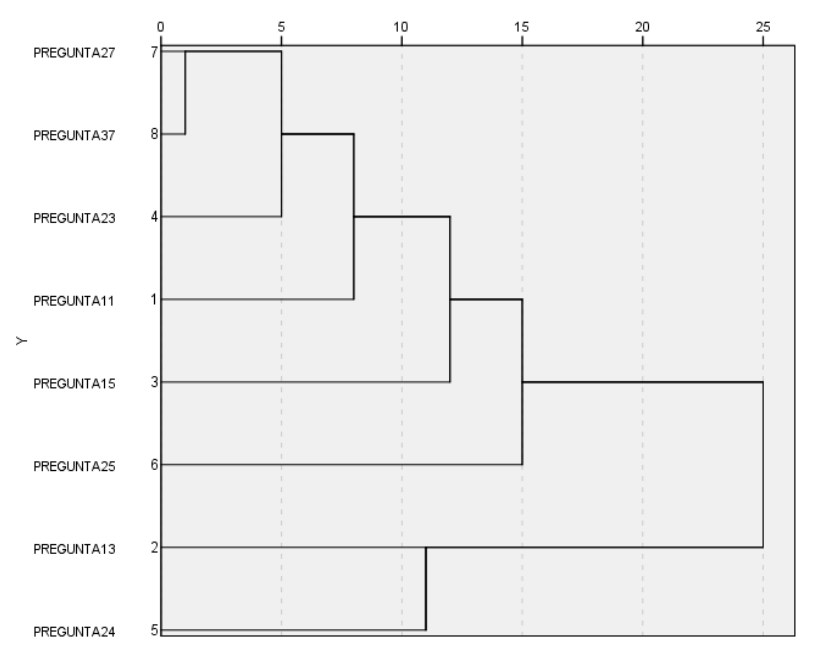

Figura 2. Dendrograma de enlace promedio, patrón estético

Preguntas

27. Considero, que aceptar la importancia del respeto por la autonomía, de la persona enferma; implica trabajar en una relación de igualdad con el enfermo y por eso dialogo con él. 37. Considero como enfermera(o); que una de las acciones más importantes de cuidado es brindar información al usuario, que es un derecho de este y una necesidad que debe ser satisfecha con eficacia. 23. En enfermería, el uso de CI escrito se debe aplicar en diferentes circunstancias: investigación, asistencia, comunidad, etc. 11. En enfermería CI, es un paso previo a la realización de todos los procedimientos, que se realizan. 15. El CI, en enfermería, solo se debe obtener cuando el procedimiento a realizar revista la presencia de daño inminente al paciente. 25. En la formación que recibí en la universidad se me enseñó sobre: ¿cómo informar?, ¿cómo dar malas noticias?, ¿cómo escuchar?, ¿cómo gestionar las dudas del paciente?; por eso NO aplico el CI, por escrito. 13. En enfermería el CI, por escrito, se le solicita al paciente siempre. 24. La existencia de un documento firmado por el paciente garantiza que éste ha comprendido la información, aun cuando se use un lenguaje técnico.

\section{Conglomerado del patrón émico}

La característica al tomar el patrón émico en el dendrograma fue de una relación marcada entre los elementos que representan "prima la vida sobre el consentimiento informado", sin importar la decisión del paciente en ese caso, sino el resguardo de la vida, y con la "creación de un ambiente para la aceptación", destacando en 
este la aprobación o el consentimiento ante las intervenciones, por lo cual esto, afirma que siempre y cuando el paciente no esté en peligro de muerte (dentro del marco de las excepciones de este consentimiento informado), puede tomarse su aprobación para procedimientos o tratamientos. Ambos aspectos resaltan las "Acciones del profesional de enfermería y los derechos del paciente", lo cual se involucra en el clúster "Aspectos operativos-comunicacionales que abarca la aplicación del CI" (Figura 3.)

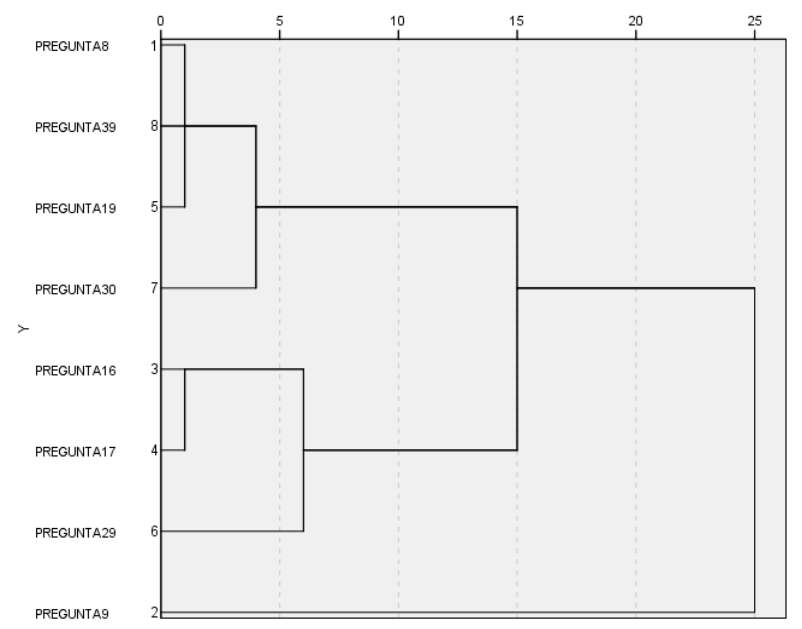

Figura 3. Dendrograma de enlace promedio, patrón émico

Preguntas

8. El CI, tiene como excepciones en su solicitud, en los casos de: urgencia, cuando está en peligro la vida del paciente, y/o cuando no dar un tratamiento represente un problema para la salud pública. 39. Considero que las intervenciones de enfermería dirigidas a menores de edad requieren el asentimiento del niño y el CI, escrito de los padres o adulto responsable del menor o el representante legal. 19. Los cuidados de enfermería relacionados con la comodidad del paciente: cambios de posición, aseo personal, administración de alimentos; requieren del CI verbal y su aceptación o rechazo por parte del paciente debe registrarse en la HC. 30. Siempre informo verbalmente al paciente sobre posibles cuidados alternativos $u$ opciones terapéuticas diferentes a las que estoy empleando en ese momento. 16. Cuando realizo un procedimiento, explico la importancia del mismo pero nunca planteo alternativas diferentes, porque sé qué el POS NO las cubre. 17. Se debe obtener CI escrito, por el paciente, únicamente cuando este decide, NO someterse al procedimiento, a realizar por el profesional de enfermería. 29. Considero, que ofrezco toda la información sobre la enfermedad cuando le informó al paciente sobre el tratamiento que le aplicarán, pero en caso de los niños esto NO es necesario. 9. Para iniciar el esquema de tratamiento de una enfermedad de salud pública como la tuberculosis, se requiere primero solicitar el CI del paciente.

\section{Conglomerado del patrón ético}

El análisis del patrón ético evidencia elementos en representación del "Código de Ética del consentimiento informado" para la atención y el respeto por el paciente, al hacer uso del instrumento, lo cual contrasta con los elementos: que el "consentimiento informado se integra a los derechos del paciente", refiriéndose a la protección de los derechos, los cuales no pueden ser violentados. Siguiendo una misma línea, se podría determinar el uso del consentimiento informado como la ética de enfermería hacia el paciente y los derechos de la persona en su posición de paciente, que se da cuando el enfermero brinda atención. Ubicándose en un clúster de categoría denominada "Derechos de los pacientes y aspectos jurídicos-legales" que afectan el CI, (Figura 4.)

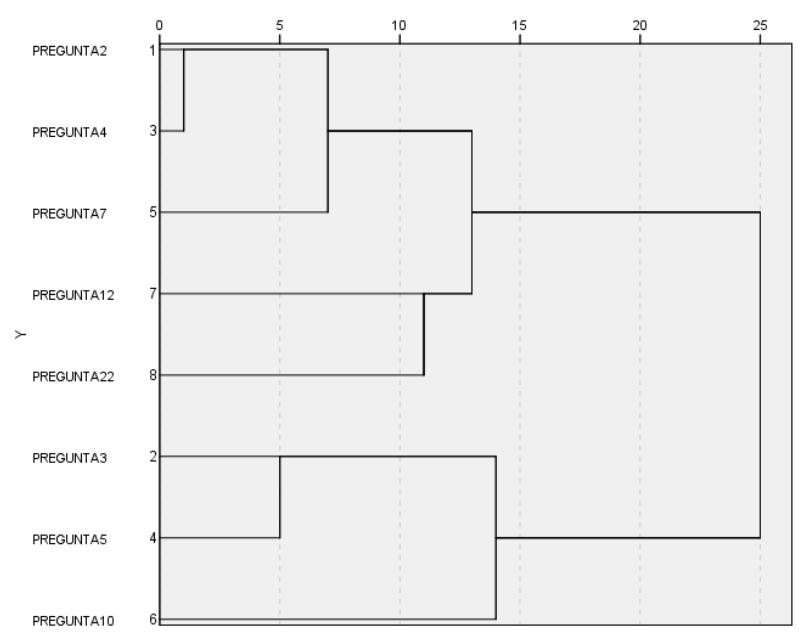

Figura 4. Dendrograma de enlace promedio, patrón ético

\section{Preguntas}

2. El CI, en enfermería, está fundamentado en el código de ética y en los principios de la bioética. 4. El CI, está integrado a los derechos y deberes del paciente, en la IPS donde laboro. 7. Cuando el paciente renuncia a recibir información, está renunciando a ejercer un derecho amparado por la ley. 12. En enfermería existe un documento denominado CI, que se usa en forma exclusiva por esta profesión. 22 . En las entidades de salud de la ciudad, existen formatos de CI escritos, que son de uso exclusivo para las intervenciones que enfermería realiza. 3. El CI, en Colombia, está más relacionado con el marco legal en salud que con la bioética. $\mathbf{5}$. El CI, es una defensa de tipo legal para las entidades de salud. 10. El uso del CI, por parte del profesional de enfermería, representa una defensa contra la mala praxis.

\section{Discusión}

\section{Análisis descriptivo}

Los datos sociodemográficos del estudio, guardan similitudes y algunas coincidencias con el estudio de tipo cuantitativo realizado en dos servicios de urgencias de España, Martín et al., encontraron que los datos demográficos evidencian que la profesión es eminentemente femenina, siendo un $70,2 \%$ de las encuestadas mujeres. No obstante, difiere en la edad promedio, con una media más alta de 35 años de edad y una experiencia de 6 años en servicios de urgencias(16).

Sobre los resultados relacionados con las instituciones por sector público y privado, fueron 
similares a los encontrados por Ovalle, en un hospital privado de Colombia y uno público de Chile, donde señala que el personal de salud no tiene mayor información sobre el consentimiento informado, y se trabaja más en función de la obtención del formato, que en el fortalecimiento de los principios bioéticos que el CI promueve. En el caso chileno no se conoce con precisión la normatividad del hospital, ni del país sobre las exigencias y cumplimiento del CI. En Colombia los médicos señalan escasez de tiempo, sobrecarga de trabajo y numeroso trámite administrativo adicional a su carga, como barreras para realizar adecuadamente el proceso de $\mathrm{CI}^{(17) .}$

\section{Análisis factorial}

La confirmación de los datos obtenidos por las puntuaciones mediados en relación a los patrones, se confirma con el análisis de varianza (ANOVA) y se concluye que no existen diferencias significativas en valores medios (en grupos etarios, género ni estudio) de los patrones: empírico, estético, émico y ético, ya que como se demostró, los valores de $\mathrm{p}$ fueron mayores a $0,05(p>0,05)$. Caso contrario, con el estudio de Bautista, donde si existen diferencias significativas en los valores medios de los patrones de conocimientos (grupos etarios, género y semestre) ${ }^{(13)}$.

Esto implica que a medida que se afianza el ejercicio profesional de enfermería, el nivel de conocimiento en el grupo de estudio, se distancia de los elementos teóricos, enfatizándose más los elementos prácticos del CI. Aspecto que concuerda a lo planteado por Núñez et al., quienes sostienen que la práctica, la teoría y la investigación son componentes básicos de la disciplina de enfermería e históricamente, la profesión se ha basado en la práctica como eje fundamental del cuidado $y$ el quehacer profesional genera procesos de fortalecimiento a partir de las experiencias, observándose el fenómeno (casual o incidental) y analizando dimensiones, por medio de la literatura e investigación, con todo esto se van adquiriendo experiencias de aprendizaje al reproducir el fenómeno y cambiar la conducta(18).
De acuerdo a lo expresado por Durán de Villalobos(11), el conocimiento empírico o ciencia de enfermería que utiliza la observación, el discurso para comunicar, los conocimientos y los datos objetivos en lo externo, se evidencia en el grupo de estudio, es decir, los profesionales saben de CI (definición), identifican su forma de representación (mediante documento escrito) y conocen su forma de difusión (explicación).

\section{Análisis inferencial por conglomerados}

Al comparar el análisis factorial e inferencial, con un estudio similar realizado con estudiantes del programa de enfermería de la UFPS, se observa que las diferencias entre conglomerados por patrones de conocimiento, no fueron muy representativas y las variaciones conceptuales encontradas pueden estar influenciadas por el contexto laboral del presente estudio(13).

\section{Conglomerado del patrón empírico}

En un estudio realizado con enfermeros de un hospital público de Chile, por León F, Burattini C, Schwartzmann J. durante el desarrollo de la entrevista se explicó a los entrevistados en qué consiste una correcta, aplicación del proceso de consentimiento informado, con el propósito de que las preguntas no fueran sesgadas por la ambigüedad que implica para cada persona el término "adecuado" [...] Con base en lo anterior, y en las respuestas obtenidas, se puede inferir que el profesional de enfermería considera que la aplicación del consentimiento informado dentro de su servicio no se realiza de manera adecuada, en lo que influye de manera considerable el factor tiempo para su realización, además del poco compromiso del equipo médico(19).

\section{Conglomerado del patrón estético}

Estos resultados concuerdan con el estudio de Galindo y Fúqene sobre el sentido y significado del $\mathrm{CI}$, en la relación de cuidado enfermerapaciente-familia, por medio del cual se indica que el tiempo es uno de los factores que influye a la hora de brindar el CI, debido a que las políticas hospitalarias alejan al profesional de enfermería de su verdadero rol, asignándole mayores funciones de tipo administrativo que en muchas ocasiones no corresponden a la profesión y le 
quita la posibilidad de estar con el usuario y su familia a la hora de ofrecer una verdadera explicación sobre las acciones de cuidado realizadas(20).

\section{Conglomerado del patrón émico}

Los resultados se asemejan al estudio de Bazán, quien refiere que la información debe ser clara, sencilla, en un lenguaje adecuado para el paciente, y con un tiempo que sea suficiente, para que esto garantice el entendimiento, además que el entorno, no sea un factor que intervenga al momento de tomarse una decisión(21).

\section{Conglomerado del patrón ético}

En un estudio descriptivo desarrollado por Iglesias et al., en un hospital universitario sobre las opiniones del personal sanitario acerca de aspectos relacionados con el consentimiento informado y muy especialmente de la información contenida en los formularios del mismo. El 45,1\% de los profesionales sanitarios consideró no tener suficiente información sobre el consentimiento informado y cuándo se debe cumplimentar por el paciente, y el $81,2 \%$ opinó que es un instrumento de protección ante demandas por parte del usuario(22), concordando con los resultados de este estudio.

\section{Conclusiones}

Las variables sociodemográficas como género, y sexo; dentro del contexto del consentimiento informado, no inciden en la importancia que el profesional de enfermería da al mismo. Se infiere que estas variables no influyen sobre los resultados del estudio. Las diferencias significativas no coexisten entre las otras variables sociodemográficas y el tipo de sector al que pertenecen las IPS, donde laboran los participantes.

En cuanto a la relación del nivel educativo y los PCE se evidencia que un mayor nivel educativo tiene efecto sobre los PCE, reflejado en los profesionales con nivel de Maestría, quienes tienen mejores conocimientos sobre el CI. Se infiere, que el nivel académico más elevado permite mejorar la episteme del conocimiento de enfermería en cuanto al manejo de CI.

En el análisis por conglomerados, surgieron nuevos clúster, los identificadores de las nuevas clasificaciones están enfocados principalmente a: derechos $y$ fundamentos procedimentales, exigencias de índole legal, administrativa y operativa al momento de realizar la gestión del CI, en los actos de la disciplina.

El nivel de conocimiento sobre el $\mathrm{CI}$, en el presente estudio no es el esperado, ya que no existe homogeneidad en las puntuaciones obtenidas en los cuatro PCI. Debido a que se evidencia incorrelación en el uso de estos patrones, tanto en el proceso como en la praxis; para el grupo de estudio.

Respecto a la importancia que los profesionales de enfermería le dan al uso del CI en los actos de cuidado de enfermería, esta se centra en los procesos legales y de defensa de los profesionales de la salud, la autorización para procesos administrativos está centrada en aspectos de auditoria, tales como: la presencia o no del documento firmado en una historia clínica, dejando en evidencia que deja de lado el componente ético y bioético del CI.

Con base en el análisis de la teoría de los patrones del conocimiento de enfermería, se identificó y se estableció cuales patrones están más favorecidos dentro del estudio, identificándose que debe promoverse el manejo adecuado del CI en los profesionales enfermería, al igual que el conocimiento sobre los elementos que lo integran, mediante el fomento de los procesos que involucran la adecuada gestión del $\mathrm{CI}$ en las instituciones de salud.

\section{Conflicto de intereses}

Ninguno declarado por los autores.

\section{Referencias}

1. Moratilla A, Cantero L, Hernández R, Molino J. La enfermería y el consentimiento informado. Cuad Bioétira [Internet]. 1998;1:64-6. Disponible en: http://aebioetica.org/revistas/1998/1/33/64.pdf

2. Asociación Colombiana de Facultades de Enfermería A. 
Noticias y eventos. In: Código de ética de enfermería Guía para el desempeño del profesional de enfermería [Internet]. Bogotá; 2006. p. 1-17. Disponible en: http://www.trienfer.org.co/index.php?option=com_co ntent\&view=article\&id=49\&Itemid $=63$

3. Alvarado A. La ética del cuidado. Rev Aquichan [Internet]. 2004;4:30-39. Disponible en: http://aquichan.unisabana.edu.co/index.php/aquichan /article/view/47/96

4. Consejo Internacional de Enfermeras. Código Deontológico del Cie para la Profesión de Enfermería [Internet]. Fornara, editor. Ginebra (Suiza); 2012. 1-12 p. Disponible en: http://publicaciones.san.gva.es/val/prof/enfermeria/d ocumentacion/codigocie2006.pdf

5. Congreso de la República de Colombia. Ley 911 de 2004, Por la cual se dictan disposiciones en materia de responsabilidad deontológica para el ejercicio de la profesión de Enfermería en Colombia; se establece el régimen disciplinario correspondiente y se dictan otras disposiciones. [Internet]. LEY 911 de 2004 Colombia: Congreso de la República; 2004 p. 15. Disponible en: http://www.mineducacion.gov.co/1621/articles105034_archivo_pdf.pdf

6. Romano G. Consentimiento informado en enfermería [Internet]. Tribunal Nacional Ético de Enfermería. 2008. p. 23. Disponible en: http://www.trienfer.org.co/index.php?option=com_co ntent\&view=article\&id=87: consentimientoinformado\&catid=35: conferencias\&Itemid $=37$

7. Rubio M, Arias M. Fenomenología y conocimiento disciplinar de enfermería. Rev Cubana Enferm [Internet]. 2013;29:191-8. Disponible en: http://scielo.sld.cu/scielo.php?script=sci_arttext\&pid= S0864-03192013000300005\&lng=es

8. Briñez KA. Narrativa de enfermería : visión y patrones de conocimiento en una entrevista en el hogar. Rev Colomb Enfermería [Internet]. 2014;9(9):142-8. Disponible

en:

http://m.uelbosque.edu.co/sites/default/files/publicac iones/revistas/revista_colombiana_enfermeria/volume n9/017-articulo15.pdf

9. Landeros A. Identificación de los patrones de conocimiento en un incidente crítico. 2002;

10. Silva MC, Sorrell JM, Sorrell CD. From Carper's patterns of knowing to ways of being: an ontological philosophical shift in nursing. ANS Adv Nurs Sci [Internet]. 1995;18(1):1-13. Disponible en: http://www.ncbi.nlm.nih.gov/pubmed/7486888

11. Duran de Villalobos MM. La Ciencia, La Etica Y El Arte En Enfermeria a Partir Del Conocimiento Personal. Rev Aquichan. 2005;5(1):86-95.

12. Soto M. Enfermería Modelo de Teselaciones para la Formación Superior [Internet]. 2nd editio. Coitinho M del C, editor. San Luis: Nueva Editorial Universitaria; 2009. 276 p. Disponible en: http://www.neu.unsl.edu.ar/wpcontent/uploads/2017/04/Enfermeria-red.pdf

13. Bautista G. Conocimiento e importancia en los estudiantes de Enfermería sobre el consentimiento informado en actos del cuidado de enfermería. Investig
Enferm Imagen Desarr [Internet]. 2015;17(2):1-17. Disponible en: http://revistas.javeriana.edu.co/index.php/imagenyde sarrollo/article/view/9528

14. Tobergte D, Curtis S. Procesamiento de Datos y Análisis Estadístico usando SPSS [Internet]. Xavier AV, editor. Vol. 53, Journal of Chemical Information and Modeling. Porto Alegre: Editora Universitária da PUCRS; 2013. 165 p. Disponible en: http://www.pucrs.br/edipucrs/spss.pdf

15. Colombia. Ministerio de Protección Social. Resolución 13437 de 19991. Congr Colomb. 1991;7-8.

16. Martín M, Ruíz F, Martín J. Consentimiento Informado En Urgencias Y Emergencias, Una Visión Real [Internet]. Cyber Revista SEEUE. 2009 [cited 2014 Nov 15]. $\quad$ p. $18 . \quad$ Disponible en: http://www.enfermeriadeurgencias.com/ciber/julio20 09/pagina6.html

17. Ovalle C. Práctica y significado del consentimiento informado en hospitales de Colombia y Chile [Internet]. Vol. 10, Revista Latinoamericana de Ciencias Sociales, Niñez y Juventud. [Bogotá]: Universidad de Manizales CINDE; Disponible en: http://revistaumanizales.cinde.org.co/index.php/Revis ta-Latinoamericana/article/view/624

18. Núñez M, Fajardo E, Quimbayo J. Situación de Enfermería Una Mirada desde los Patrones, de Conocimiento y el Proceso de Atención [Internet]. Revista de Actualizaciones en Enfermería. 2011 [cited 2014 Jul 12]. p. 4. Disponible en: https://encolombia.com/medicina/revistasmedicas/enfermeria/ve-144/situaciondeenfermeria/

19. León J, Burattini C, Schwartzmann J. El consentimiento informado y el profesional de enfermería: estudio cualitativo en hospital público de Chile. rev eleuthera [Internet]. 2009;3:221-37. Disponible en: http://200.21.104.25/eleuthera/downloads/Eleuthera 3_10.pdf

20. Fuquene J, Galindo D. Sentido y significado del consentimiento informado en la relación de cuidado enfermera-usuario-familia [Internet]. Vol. 3, Biblioteca Lascasas. Universidad Pedagógica y Tecnológica de Colombia; 2007. Disponible en: http://www.indexf.com/lascasas/documentos/lc0196.pdfv

21. Bazán A. Experiencias de las enfermeras sobre el consentimiento informado en el cuidado a personas hospitalizadas en servicios críticos Chiclayo, Perú [Internet]. Universidad Católica Santo Toribio de Mogrovejo; 2012. Disponible en: http://tesis.usat.edu.pe/bitstream/usat/596/1/TM_Ba zan_Sanchez_Asuncion.pdf

22. Iglesias Lepine ML, Botet- Montoya J, Pallás Villaronga O, Hernández Leal E, Echarte JL, Solsona Durán JF. Consentimiento informado: opiniones del personal sanitario de un hospital universitario. Rev Clínica Española [Internet]. 2007;207(10):483-8. Disponible en:http://www.sciencedirect.com/science/article/pii/ S0014256507734550 